\title{
Dormancy and spring burst of lateral buds on stems of low-pruned mulberry (Morus alba L.)
}

\author{
T. Suzuki ${ }^{1}$, M. Kitano ${ }^{2}$ and K. Kohno² \\ 1 Faculty of Textile Science, Kyoto Kogei-Sen-i University, Matsugasaki, Kyoto 606, and \\ 2 Faculty Farmstead, Kyoto Kogei-Sen-i University, Saga, Kyoto 616, Japan
}

\section{Introduction}

After decapitation, lateral shoot growth of mulberry coppice (Morus alba L.) from low-pruned stumps has an extremely acrotonic form, in which only a few upper buds grow out to form branches (Suzuki et al., 1988). In contrast, the majority of the buds of 1 yr old stems, including the basal buds, burst into growth almost simultaneously and elongate in the spring (Suzuki and Kohno, 1987).

Bud dormancy and the release of lateral buds from dormancy are related in some way to the stem and to the location of these buds on the stem, and greatly affect the pattern of bud development in the new growth of trees in the spring (Meng Horn et al., 1975; Crabbé, 1981, 1984a, b). Although many experiments with exogenous and/or endogenous growth inhibitors and growth promoters have been conducted on the control of bud dormancy as well as bud development, less attention has been devoted to lateral buds located along the stem in dormancy research. This study was undertaken to define the role of lateral bud dormancy on spring burst and shoot growth in the low-pruned mulberry.

\section{Materials and Methods}

Rooted hardwood cuttings of Morus alba L. CV. Shin-ichinose were grown in a light clay soil at the Saga Experimental Farm of Kyoto KogeiSen-i University, Kyoto, Japan. The cuttings were produced in the spring of 1977 and pruned annually from 1979 to 1986 to maintain stump heights at $40-50 \mathrm{~cm}$ (in early March). The dominant coppice shoots were allowed to grow from 1986 through 1987 and used for the experiments (Fig. 1).

Twenty shoots (about $210 \mathrm{~cm}$ long) were collected at 2-5 wh intervals from 25 August to 16 March (Fig. 2). After harvesting and, when necessary, defoliation, these stems were divided into 5 regions and the segments (length about $15 \mathrm{~cm}$ ) to be tested were collected from the upper portion of each region of the stems, respectively (Fig. 1). About $10 \mathrm{~cm}$ of the stem apices were excluded from the experiments because the majority of the uppermost buds usually could not grow out into shoots (Suzuki and Kohno, 1987). Unless otherwise stated, 5 groups of 20 segments each were then incubated in water to a depth of $c a 1.5 \mathrm{~cm}$ at $27^{\circ} \mathrm{C}$ in the dark, and the subsequent bud break of the segments was recorded. Buds were considered 


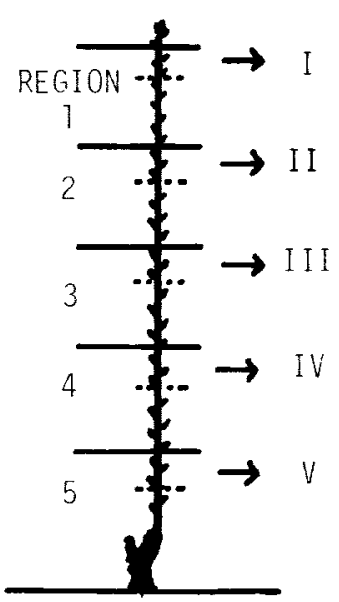

Fig. 1. Diagram of the sampling plan used and sample identification. Only the treated coppice shoot on each stump is illustrated, the others are omitted.

'sprouting' when the bud scales had separated and the tips of developing leaves were visible. The incubating water was changed every other day during the incubation period.

\section{Results}

When studied by a multiple-node-cutting test (Fig. 2), the buds of the upper segment 1 experienced their maximum depth of dormancy around November and December. In contrast, buds of the lower segments were less dormant than those of the upper segment until February, and there was $100 \%$ breaking of the buds of segment $V$ throughout. In August and September, unlike the extremely acrotonic growth habit of the decapitated shoots (Suzuki et al., 1988), the stems showed a clear basitonic gradient in bud growth potential, in which the lower buds grew out faster than the upper ones. This basitonic gradient persisted until November, when dormancy of the upper buds reached its peak. The gradient then weakened and changed towards a linear one, but not an acrotonic one, with the disappearance of the dormant condition in February and March.

\section{Discussion and Conclusion}

In trees, lateral buds on the previous year's growth are released from correlative inhibition by a period of winter dormancy. This release may certainly be related to dormancy gradient changes between buds along the stem, which induce an acrotonic gradient in the new growth of trees in the spring (Meng Horn et al., 1975; Crabbé, 1981, 1984a, b). In lowpruned mulberry, however, the gradient changes toward a linear one, with a disappearance of the dormant condition in February and March (Fig. 2). As a result, bud burst and shoot growth in low-pruned mulberry occur readily from the majority of buds, including the basal buds, in the spring.

\section{Acknowledgments}

We thank Mr. H. Fujiwara of the Saga Experimental Farm, Kyoto Kogei-Sen-i University, Kyoto, Japan, for maintenance of the cultivated mulberry trees.

\section{References}

Crabbé J.J. (1981) The influence of bud dormancy in the morphogenesis of trees and shrubs. Acta Hortic. 120, 167-172

Crabbé J.J. (1984a) Morphogenetical ways towards vigor restriction in spontaneous and 

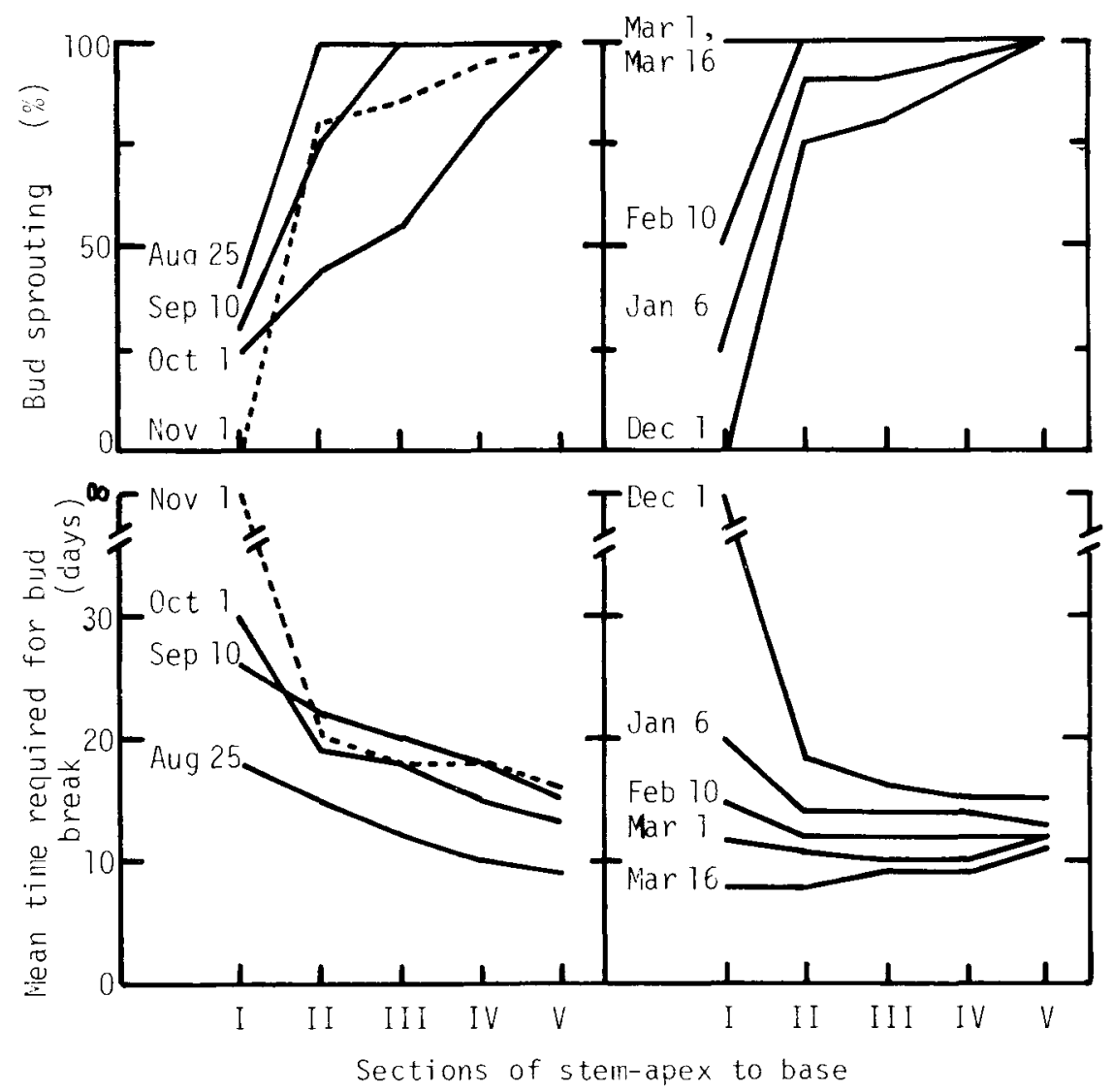

Fig. 2. Bud growth potential gradients observed in mulberry. Sampling date is given on each graph. Samples from August 26 and September 10 were incubated in water at room temperature under natural day length. The segments, $I-V$, as in Fig. 1.

man-made dwarf trees. Acta Hortic. 146, 113120

Crabbé J.J. (1984b) Correlative effects modifying the course of bud dormancy in woody plants. Z. Pflanzenphysiol. 113, 465-469

Meng Horn C., Champagnat P., Barnola P. \& Lavarenne S. (1975) L'axe caulinaire, facteur de préséances entre bourgeons, sur le rameau de l'année du Rhamnus frangula L. Physiol. Vég. 13, 335-348

Suzuki T., Kitano M. \& Kohno K. (1988) Lateral bud outgrowth on decapitated shoots of lowpruned mulberry (Morus alba L.). Tree Physiol. $4,53-60$ 